\section{C BUILDING CAPACITIES ON OSH IN DEVELOPING COUNTRIES: EXPERIENCES FROM FINLAND}

${ }^{1} S$ Lehtinen*, ${ }^{2}$ J Rantanen. ${ }^{1} \mathrm{MSC}$ (Econ.), Tampere, Finland; ${ }^{2}$ Helsinki University, Department of Public Health/Occupational Health, Helsinki, Finland

\subsection{6/oemed-2018-ICOHabstracts.873}

Introduction The socio-economic development of any country is dependent on the health, safety and well-being of its working population. In its development collaboration projects over the years, Finland has prioritised the improvement of working conditions and thereby the prevention of occupational accidents and diseases. Occupational health and safety collaboration has taken place with East Africa in particular, but also with South-East Asian countries.

Method Collaboration began as the provision of training for $\mathrm{OSH}$ experts, and later evolved into either country-targeted or regional programmes for developing the $\mathrm{OSH}$ infrastructures of the countries. These programmes included the capacity building of the actors involved. The practical collaboration with East Africa was realised together with the OSH Inspectorates, and the Institutes of Occupational Health in the countries, in which these were available. The financial support of the Finnish Ministry for Foreign Affairs made it possible to run the programmes.

Results In 1973-2009, collaboration grew from individual training courses for OSH experts in Zambia, Tanzania and later in Kenya, to more comprehensive projects that covered legislation development, policy and strategy planning, and support for practice-oriented research, training, information dissemination, and the provision of services. In the 1980s, the ILO-run Strengthening of Factories Inspectorates projects introduced a wider approach to OSH activities. At the end of the 1980s an East African Regional OSH programme was carried out with follow-up symposia in 1992, 1995 and 1998. Later in 2008-2009, a regional follow-up programme on OSH was conducted in East Africa. Many of the trainees of the time were or are now in key senior positions in occupational health and safety administration or other relevant organisations, including enterprises.

Discussion Among the most important features of collaboration and the lessons learnt during this are the comprehensive content of collaboration; maximising the use of networking, internationally, nationally and locally; and ensuring the continuity of the activities. This means that a committed staff is essential. The long-term development impact of expert training and training of future $\mathrm{OH}-\mathrm{OSH}$ leaders was deemed highly positive.

\section{2d CAPACITY BUILDING IN OSH: A VIEW FROM THE DEVELOPING COUNTRY}

${ }^{1} \mathrm{~S}$ Pingle ${ }^{*},{ }^{2} \mathrm{R}$ Parekh, ${ }^{1} \mathrm{D}$ Mavalankar. ${ }^{1}$ Indian Institute of Public Health Gandhinagar, India; ${ }^{2}$ Occupational Health Consultant, Mumbai, India

\subsection{6/oemed-2018-ICOHabstracts.874}

Access to specialised occupational health services is low across the world. However, the problem is very acute in developing countries due to various factors. Equitable health system cannot be achieved unless the lack of basic occupational health facilities for all the working population are addressed. India is an important and major developing country and represents the challenges in the developing countries to a great extent.

The most important $\mathrm{OSH}$ needs in developing countries such as India are:

1. legislation to extend $\mathrm{OSH}$ coverage to all sectors of working life including the unorganised sector,

2. spreading the awareness about OSH,

3. development of OSH infrastructure and OSH professionals.

4. Integration of occupational health with primary health care.

To address the increasing need of occupational health experts, capacity building is needed on a massive scale. The status of occupational health capacity in India reviewed from the available data and literature indicates huge gaps in the requirement and available capacity. The capacity established by the government has been grossly inadequate. Further, currently the services are aimed at organised sector and the huge need in unorganised sector is not being addressed.

Various nongovernmental international and national organisations have stepped in and have initiated various sensitisation, awareness and training programs with support from donors. Some of these programs have supported training of few experts from developing countries including India.

A case study of occupational health training and sensitisation of health care providers at primary level will be discussed. It is an initiative by Indian Association of Occupational Health which is a nongovernmental voluntary professional organisation of $\mathrm{OH}$ experts. Two of the international programs will also be discussed.

This presentation will review some of these initiatives and their outcome, the challenges faced and way forward. It will also discuss the potential role of expert associations.

\section{2e PROJECT-BASED TRAINING AS A TOOL TO TACKLE OCCUPATIONAL HEALTH CHALLENGES IN DEVELOPING COUNTRIES}

${ }^{1,2} \mathrm{~K}$ Radon ${ }^{*},{ }^{1,2} \mathrm{~L}$ Kurtz, ${ }^{1,2} \mathrm{D}$ Carvalho, ${ }^{1,2} \mathrm{~V}$ Encina, ${ }^{1,2} \mathrm{~F}$ van Dijk, ${ }^{1,2} \mathrm{C}$ Meneses, 1,2MA Garrido, 1,2MF Bauleo, 1,2 L Briceño, ${ }^{1,2} \mathrm{R}$ Herrera, ${ }^{1,2} \mathrm{M}$ Parra. ${ }^{1}$ Center for International Health LMU @ Institute and Clinic for Occupational, Social and Environmental Medicine, University Hospital, LMU Munich, Germany; ${ }^{2}$ Supported by German Academic Exchange Service, DAAD, via its Exceed program funded by the German Federal Ministry for Economic Cooperation and Development

\subsection{6/oemed-2018-ICOHabstracts.875}

Introduction To know the prevalence of exposures and diseases at workplaces is crucial for the development of intervention strategies and healthcare planning. However, in many low and middle-income countries (LMIC) research is no priority. One reason is a lack of experts able to carry out sound epidemiological research at workplaces. One way of preparing such experts is through project-based training. We therefore implemented a project as training method in our blended learning Master in International Occupational Safety and Health $(\mathrm{OSH})$.

Methods Students start by identifying their research interest based on existing worker's health problems. They develop a study protocol to assess working conditions and health at a self-selected workplace. After ethics approval, they carry out the project during the 2 nd term of the program. In term 3 , they learn to analyse and interpret data and develop a teaching intervention. In the final term, students write the master 